Groups Geom. Dyn. 7 (2013), 867-882

DOI $10.4171 / \mathrm{GGD} / 208$
Groups, Geometry, and Dynamics

(C) European Mathematical Society

\title{
On the product decomposition conjecture for finite simple groups
}

\author{
Nick Gill, László Pyber, Ian Short and Endre Szabó*
}

\begin{abstract}
We prove that if $G$ is a finite simple group of Lie type and $S$ is a subset of $G$ of size at least two, then $G$ is a product of at most $c \log |G| / \log |S|$ conjugates of $S$, where $c$ depends only on the Lie rank of $G$. This confirms a conjecture of Liebeck, Nikolov and Shalev in the case of families of simple groups of bounded rank. We also obtain various related results about products of conjugates of a set within a group.
\end{abstract}

Mathematics Subject Classification (2010). 20D06, 20D40, 20 G40.

Keywords. Conjugacy, Doubling Lemma, Product Theorem, simple group, width.

\section{Introduction}

Our starting point is the following conjecture of Liebeck, Nikolov and Shalev [13].

Conjecture 1.1. There exists an absolute constant $c$ such that if $G$ is a finite simple group and $S$ is a subset of $G$ of size at least two, then $G$ is a product of $N$ conjugates of $S$ for some $N \leq c \log |G| / \log |S|$.

Note that we must have $N \geq \log |G| / \log |S|$ by order considerations, and so the bound above is best possible up to the value of the constant $c$.

The conjecture is an extension of a deep (and widely applied) theorem of Liebeck and Shalev [16]. Indeed, the main result of [16] states that the above conjecture holds when $S$ is a conjugacy class or, more generally, a normal subset (that is, a union of conjugacy classes) of $G$ (we state this result formally in Theorem 5.1). In [13] Conjecture 1.1 is also proved for sets of bounded size.

Somewhat earlier Liebeck, Nikolov and Shalev [11] posed the following (still unproved) weaker conjecture.

${ }^{*}$ L. P. is supported in part by OTKA NK78439 and K84233. E. Sz. is supported in part by OTKA NK81203 and K84233. N. G. would like to thank Harald Helfgott for allowing the use of his research funds to facilitate a visit to the Rényi Institute during which work on this paper was initiated. He would also like to thank the University of Bristol to which he has been a regular visitor over the course of writing this paper. 
Conjecture 1.2. There exists an absolute constant $c$ such that if $G$ is a finite simple group and $H$ is any nontrivial subgroup of $G$, then $G$ is a product of $N$ conjugates of $H$ for some $N \leq c \log |G| / \log |H|$.

Conjecture 1.2 itself represents a dramatic generalization of a host of earlier work on product decompositions of finite simple groups, most of which prove Conjecture 1.2 for particular subgroups $H$. For instance, in [15] it is proved that a finite simple group of Lie type in characteristic $p$ is a product of 25 Sylow $p$-subgroups (see also [1] for a recent improvement from 25 to 5).

Further positive evidence for Conjecture 1.2 is provided by [12], [17] and [18] (when $H$ is of type $\mathrm{SL}_{n}$ ). Certain results of this type are essential to prove that finite simple groups can be made into expanders (see the announcement [8]).

The main purpose of this note is to prove Conjecture 1.1 for finite simple groups of Lie type of bounded rank. Put another way, we prove a version of Conjecture 1.1 in which the constant $c$ depends on the rank of the group $G$. Our main result follows.

Theorem 1.3. Fix a positive integer $r$. There exists a constant $c=c(r)$ such that if $G$ is a finite simple group of Lie type of rank $r$ and $S$ is a subset of $G$ of size at least two, then $G$ is a product of $N$ conjugates of $S$ for some $N \leq c \log |G| / \log |S|$.

In [13] a weaker bound of the form $N \leq(\log |G| / \log |S|)^{c(r)}$ is obtained. Also, in [11], Theorem 1.3 is proved when $S$ is a maximal subgroup of $G$.

As a byproduct of our proof we obtain two results of independent interest. In these results, and throughout the paper, we denote by $S^{g}$ the conjugate $g^{-1} S g$ of a subset $S$ of a group $G$ by an element $g$ of $G$, and, given a positive integer $m$, we denote by $S^{m}$ the product $S S \ldots S$ of $m$ copies of $S$. There should be no confusion between these two similar notations because the type of the exponent will always be given.

Theorem 1.4. Fix a positive integer $r$. There exists a positive constant $\varepsilon=\varepsilon(r)$ such that if $G$ is a finite simple group of Lie type of rank $r$ and $S$ is a subset of $G$ then either $\left|S S^{g}\right| \geq|S|^{1+\varepsilon}$ for some $g$ in $G$ or $S^{3}=G$.

The next theorem is similar, but concerns only normal subsets, in which case we obtain absolute constants.

Theorem 1.5. There exists $\varepsilon>0$ and a positive integer $b$ such that if $G$ is a finite simple group and $S$ is a normal subset of $G$ then either $\left|S^{2}\right| \geq|S|^{1+\varepsilon}$ or $S^{b}=G$.

Theorem 1.5 relates to a result of Shalev, Theorem 7.4 of [27], which we strengthen in Section 5.

Note that Theorem 1.5 would not be true were we to consider sets that are not normal. For instance, take $S$ to be a maximal parabolic subgroup in $G=\operatorname{PSL}_{n}(q)$ 
with index $\frac{q^{n}-1}{q-1}$. Clearly $S^{b}=S$ for all positive integers $b$; on the other hand, for any positive number $\varepsilon$, and any $g$ in $G$, we have $\left|S S^{g}\right| \leq|G|<|S|^{1+\varepsilon}$ once $n$ is large enough. We conclude that neither of the given options can hold in this more general situation.

Theorems 1.4 and 1.5, and the remarks of the previous paragraph, lead us to make the following conjecture.

Conjecture 1.6. There exists $\varepsilon>0$ and a positive integer $b$ such that if $S$ is a subset of a finite simple group $G$, then for some $g$ in $G$ either $\left|S S^{g}\right| \geq|S|^{1+\varepsilon}$ or $G$ is the product of $b$ conjugates of $S$.

Note that, by Theorems 1.3 and 1.4, Conjectures 1.1, 1.2 and 1.6 hold for all exceptional simple groups. Note too that all three conjectures could be phrased in terms of translates of the set $S$, rather than conjugates. This follows from the simple fact that a product of translates of $S$ is equal to a translate of a product of conjugates of $S$. Similarly a product of conjugates of a translate of $S$ is equal to a translate of a product of conjugates of $S$, a fact which will be useful in its own right.

It is possible that Conjecture 1.6 actually holds with $b=3$. When $b=2$ counterexamples are given by large non-real conjugacy classes (see the final section of [27] for some related issues). Further counterexamples are given by certain families of maximal subgroups (see for example Corollary 2 of [14], which states that large enough simple unitary groups of odd dimension cannot be decomposed into the product of two proper subgroups).

We derive Theorems 1.3 and 1.4 as consequences of the recent Product Theorem for finite simple groups, proved independently by Breuillard, Green and Tao [3], and Pyber and Szabó [23] (see Section 2). Theorem 1.5 follows from a version of Conjecture 1.1 for normal subsets due to Liebeck and Shalev [16] and an extension of Plünnecke's theorem, Theorem 6.27 of [30], to normal subsets of nonabelian groups (see Section 4).

In the final section we use a result of Petridis [20] to derive an analogue of the classical Doubling Lemma, a special case of Plünnecke's theorem. We refer to the new result as the Skew Doubling Lemma; it can be thought of as a nonabelian version of the classical Doubling Lemma. The Skew Doubling Lemma is applied to prove that Conjecture 1.1 implies Conjecture 1.6. In the other direction, a standard argument (similar to the proof of Corollary 2.8) shows that Conjecture 1.6 implies that a simple group $G$ is a product of $(\log |G| / \log |S|)^{c}$ conjugates of $S$, a weaker version of Conjecture 1.1.

\section{Proof of Theorem 1.4}

We begin with a result of Petridis, Theorem 4.4 of [20], which extends work of Helfgott, Ruzsa and Tao [7], [25], [26], [29]. It relates to the Doubling Lemma for abelian groups, which we return to in Section 4. 
Lemma 2.1. Let $S$ be a finite subset of a group $G$. Suppose that there exist positive numbers $J$ and $K$ such that $\left|S^{2}\right| \leq J|S|$ and $|S g S| \leq K|S|$ for each $g$ in $S$. Then $\left|S^{3}\right| \leq J^{7} K|S|$.

Suppose now that $G$ is a finite group. Let $\operatorname{minclass}(G)$ denote the size of the smallest nontrivial conjugacy class in $G$. Given a subset $S$ of $G$ that is neither empty nor the trivial group, we denote by minclass $(S, G)$ the size of the smallest nontrivial conjugacy class in $G$ that intersects $S$. Finally, let $\operatorname{mindeg}(G)$ denote the dimension of the smallest nontrivial complex irreducible representation of $G$.

As observed in [19], a result of Gowers [4] implies the following.

Proposition 2.2. Let $G$ be a finite group and let $k=\operatorname{mindeg}(G)$. Take $S \subseteq G$ such that $|S| \geq \frac{|G|}{\sqrt[3]{k}}$. Then $G=S^{3}$.

Now let $G=G_{r}(q)$ be a simple group of Lie type of rank $r$ over $\mathbb{F}_{q}$, the finite field of order $q$. We need some facts about $G$. The first result can be deduced, for example, from Tables 5.1 and Theorem 5.2.2 of [9].

Proposition 2.3. We have $q^{r} \leq \operatorname{minclass}(G)<|G| \leq q^{8 r^{2}}$.

Proposition 2.4. Let $k=\operatorname{mindeg}(G)$. Then $|G|<k^{8 r^{2}}$.

Proof. We use the lower bounds on projective representations given by Landazuri and Seitz [10], allowing for the slight errors corrected in Table 5.3.A of [9]. For $G \neq \operatorname{PSL}_{2}(q)$, we see that $k \geq q$, and so the result follows from Proposition 2.3.

Now suppose that $G=\operatorname{PSL}_{2}(q)$; then $|G|<q^{3}$ and $r=1$. For $q \geq 5$ and $q \neq 9, k=\frac{1}{(2, q-1)}(q-1)$ and it is clear that $k^{8}>q^{3}$. When $q=4$ we have $k=2$ and the result follows; likewise when $q=9$ we have $k=3$ and the result follows.

The next result was obtained independently in [5] and [28].

Proposition 2.5. Each finite simple group $G$ is $\frac{3}{2}$-generated; that is, for any nontrivial element $g$ of $G$ there exists $h$ in $G$ such that $\langle g, h\rangle=G$.

Corollary 2.6. Let $G$ be a finite simple group and let $S$ be a subset of $G$ of size at least two. Then some translate of $S$ generates $G$.

Proof. Let $u$ and $v$ be distinct elements of $S$. Since $G$ is $\frac{3}{2}$-generated, there exists $x$ in $G$ such that $\left\langle v u^{-1}, x\right\rangle=G$. Therefore the translate $S u^{-1} x$, which contains $x$ and $v u^{-1} x$, generates $G$.

The next result, the Product Theorem, is our primary tool for proving Theorems 1.3 and 1.4. Versions of this result can be found in [3], [23]. It was first proved by Helfgott for the groups $\mathrm{PSL}_{2}(p)$ and $\mathrm{PSL}_{3}(p)$ in [6], [7]. 
Theorem 2.7. Fix a positive integer $r$. There exists a positive constant $\eta=\eta(r)$ such that, for $G$ a finite simple group of Lie type of rank $r$ and $S$ a generating set of $G$, either $S^{3}=G$ or $\left|S^{3}\right| \geq|S|^{1+\eta}$.

We can now prove Theorem 1.4.

Proof of Theorem 1.4. Given a positive integer $r$, let $\eta$ be the constant from Theorem 2.7. Let $L=8^{\frac{2}{n}}$.

Suppose first that $|S| \leq L$. The result holds trivially if $S=G$ or $|S| \leq 1$, so let us assume that $S \neq G$ and $|S|>1$. We will show that there is an element $g$ such that $\left|S S^{g}\right| \geq|S|+1$. By replacing $S$ with a translate, we may assume that $S$ contains the identity. Therefore $S^{2} \supseteq S$. If the inequality $\left|S^{2}\right| \geq|S|+1$ does not hold then instead $\left|S^{2}\right|=|S|$, so $S^{2}=S$, and hence $S$ is a group. It is not a normal subgroup (because $G$ is simple) so there are elements $x$ and $g$ in $G$ with $x \in S^{g}$ but $x \notin S$. Then $S S^{g} \supseteq S \cup\{x\}$, so $\left|S S^{g}\right| \geq|S|+1$, as required. Let $\varepsilon_{1}=\log (L+1) / \log L-1$. One can check that $|S|+1 \geq|S|^{1+\varepsilon_{1}}$, and hence there is an element $g$ of $G$ such that $\left|S S^{g}\right| \geq|S|^{1+\varepsilon_{1}}$.

Suppose now that $|S|>L$. Since $G$ is $\frac{3}{2}$-generated, there exists an element $g$ of $G$ such that the set $T=S \cup\{g\}$ generates $G$. We can apply Theorem 2.7 to $T$ to conclude that either $\left|T^{3}\right| \geq|S|^{1+\eta}$ or $T^{3}=G$. We consider each possibility in turn.

Suppose that $\left|T^{3}\right| \geq|S|^{1+\eta}$. Observe that $T^{3}$ is the union of the eight sets $S S S, S S g, S g S, g S S, S g g, g S g, g g S$ and $\{g g g\}$. Therefore at least one of the eight sets is larger than $\frac{1}{8}|S|^{1+\eta}$. We assumed earlier that $|S|>8^{\frac{2}{\eta}}$, from which it follows that $\frac{1}{8}|S|^{1+\eta}>|S|^{1+\frac{\eta}{2}}$. Therefore one of the first seven of the eight sets is larger than $|S|^{1+\frac{\eta}{2}}$. All of these seven sets except $S S S$ are equal to a translate of the product of one or two conjugates of $S$, so if any of these have size at least $|S|^{1+\frac{\eta}{2}}$ then $\left|S S^{h}\right| \geq|S|^{1+\frac{\eta}{2}}$ for some element $h$ of $G$. If, on the other hand, $|S S S|>|S|^{1+\frac{n}{2}}$, then Lemma 2.1 (with $J=K=|S|^{\frac{n}{16}}$ ) implies that there is an element $h$ of $S \cup\{1\}$ with $\left|S S^{h}\right| \geq|S|^{1+\frac{\eta}{16}}$. Therefore in both cases there is an element $h$ with $\left|S S^{h}\right| \geq|S|^{1+\varepsilon_{2}}$, where $\varepsilon_{2}=\frac{\eta}{16}$.

The remaining possibility is that $T^{3}=G$. If $S^{3} \neq G$ then Proposition 2.2 implies that $|S| \leq|G| / \sqrt[3]{k}$ where $k=\operatorname{mindeg}(G)$. But Proposition 2.4 gives that $|S| \leq|G|^{1-\frac{1}{24 r^{2}}}$, and this implies, in particular, that $\left|T^{3}\right|=|G| \geq|S|^{1+\frac{1}{24 r^{2}}}$. The argument of the previous paragraph applies again, to give a positive constant $\varepsilon_{3}$ that depends only on $r$ such that $\left|S S^{h}\right| \geq|S|^{1+\varepsilon_{3}}$ for some element $h$.

Let $\varepsilon$ be the minimum of $\varepsilon_{1}, \varepsilon_{2}$ and $\varepsilon_{3}$; this depends only on $r$. We have shown that if $S^{3} \neq G$ then there is an element $g$ of $G$ with $\left|S S^{g}\right| \geq|S|^{1+\varepsilon}$. This completes the proof.

Note that we can immediately deduce the following result of [13] (which we will use later). 
Corollary 2.8. Fix a positive integer $r$. There exists a constant $d$ such that if $G$ is a finite simple group of Lie type of rank $r$ and $S$ is a subset of $G$ of size at least two, then $G$ is a product of $N$ conjugates of $S$ for some $N \leq 3(\log |G| / \log |S|)^{d}$.

Proof. Let $\varepsilon$ be the constant from Theorem 1.4, and define $d=\log _{1+\varepsilon} 2$. Let $M$ be the integer part of $\log _{1+\varepsilon} \frac{\log |G|}{\log |S|}$. Theorem 1.4 implies that $G$ is the product of $3 \cdot 2^{M}$ conjugates of $S$, and

$$
3 \cdot 2^{M} \leq 3\left(\frac{\log |G|}{\log |S|}\right)^{d} .
$$

The results in this section motivate a common generalisation of the Product Theorem (that is, Theorem 2.7) and Conjecture 1.6 for groups of Lie type.

Conjecture 2.9. There exists $\varepsilon>0$ and a positive integer $b$ such that the following statement holds. For each positive integer $r$ there is a positive integer $c(r)$ such that if $G$ is a finite simple group of Lie type of rank $r$ and $S$ a generating set of $G$, then either $\left|S S^{g}\right| \geq|S|^{1+\varepsilon}$ for some $g \in S^{c(r)}$, or else $G$ is the product of $b$ conjugates $S^{g_{1}}, \ldots, S^{g_{b}}$, where $g_{1}, \ldots, g_{b} \in S^{c(r)}$.

It would be interesting to prove Conjecture 1.6 in the case when $S$ is a subgroup of $G$. A rather general qualitative result in this direction was obtained by Bergman and Lenstra [2]. They show that if $H$ is a subgroup of a group $G$ satisfying $\left|H H^{g}\right| \leq$ $K|H|$ for all $g$ in $G$, then $H$ is "close to" some normal subgroup $N$ of $G$, in the sense that $|H: H \cap N|$ and $|N: H \cap N|$ are both bounded in terms of $K$.

\section{Proof of Theorem 1.3}

Given an element $g$ of a group $G$ we define

$$
g^{G}=\left\{g^{h} \mid h \in G\right\},
$$

and, for a subset $Z$ of $G$,

$$
Z^{G}=\left\{Z^{h} \mid h \in G\right\} .
$$

We begin the proof of Theorem 1.3 with a simple combinatorial lemma, which enables us to deal with "small" sets.

Lemma 3.1. Let $S$ be a subset of size at least two in a finite group $G$. There exist a positive integer $m$ and a set of $m$ conjugates of $S$ whose product $X$ satisfies

$$
|X|=|S|^{m} \geq \frac{\sqrt{\operatorname{minclass}\left(S S^{-1}, G\right)}}{|S|} \geq \frac{\sqrt{\operatorname{minclass}(G)}}{|S|} .
$$


Proof. Define $X_{1}=S$ and, if possible, choose an element $g$ of $G$ such that $X_{1}^{-1} X_{1} \cap$ $g S S^{-1} g^{-1}=\{1\}$. Define $X_{2}=X_{1} g S g^{-1}$. Notice that if $x_{L}, x_{R} \in X_{1}, s_{L}, s_{R} \in$ $S$, and $x_{L} g s_{L} g^{-1}=x_{R} g s_{R} g^{-1}$, then $x_{R}^{-1} x_{L}=g s_{R} s_{L}^{-1} g^{-1}$. Hence $x_{R}^{-1} x_{L} \in$ $X_{1}^{-1} X_{1} \cap g S S^{-1} g^{-1}$, and so $x_{L}=x_{R}$ and $s_{L}=s_{R}$. It follows that $\left|X_{2}\right|=\left|X_{1}\right||S|$. Now repeat this process with $X_{2}$ replacing $X_{1}$, and so on.

The process terminates with a set $X$ of size $|S|^{m}$, which is a product of $m$ conjugates of $S$, and such that $\left|X^{-1} X \cap g S S^{-1} g^{-1}\right| \geq 2$ for all $g$ in $G$.

Let $T$ be a set of smallest possible size that intersects every conjugate of $Z=$ $S S^{-1}$ nontrivially, and write $t=|T|$. Let $n=\left|G: N_{G}(Z)\right|$, the number of $G$ conjugates of $Z$. By the pigeonhole principle there exists an element $g$ of $Z$ that lies in at least $\frac{n}{t}$ different conjugates of $Z$. Let us count the set

$$
\Omega=\left\{\left(g^{\prime}, Z^{\prime}\right) \in g^{G} \times Z^{G} \mid g^{\prime} \in Z^{\prime}\right\}
$$

in two different ways.

First, since every conjugate of $g$ lies in the same number of conjugates of $Z$, we know that $\left|g^{G}\right| \frac{n}{t} \leq|\Omega|$. On the other hand it is clear that $|\Omega| \leq n|Z|$. Putting these together we obtain that $\left|g^{G}\right| \frac{n}{t} \leq n|Z|$. Therefore

$$
t \geq \frac{\left|g^{G}\right|}{|Z|} \geq \frac{\operatorname{minclass}\left(S S^{-1}, G\right)}{|S|^{2}}
$$

and using $|X|^{2} \geq\left|X^{-1} X\right| \geq t$ our statement follows.

Remark 3.2. Lemma 3.1 and Proposition 2.3 imply that if $G$ is a simple group of Lie type of rank $r$ and $S$ is a subset of size less that $q^{r / 4}$, then we have $\left|S S^{g}\right|=|S|^{2}$ for some $g$ in $G$.

We are now ready to prove Theorem 1.3.

Proof of Theorem 1.3. As observed above, a product of conjugates of a translate of $S$ is equal to the translate of a product of conjugates of $S$. By Corollary 2.6, a translate of $S$ generates $G$. Therefore we assume that $S$ generates $G$.

Suppose that $|S| \geq \operatorname{minclass}(G)^{1 / 4}$; then $|G|<|S|^{32 r}$ by Proposition 2.3. Now Corollary 2.8 implies that $G$ is a product of fewer than $3(32 r)^{d}$ conjugates of $S$. The theorem holds in this case with $c=3(32 r)^{d}$.

Suppose instead that $|S|<\operatorname{minclass}(G)^{1 / 4}$. By Lemma 3.1 we can choose conjugates $S_{1}, \ldots, S_{m}$ of $S$ such that the set $X=S_{1} \ldots S_{m}$ satisfies $|X|=|S|^{m}$ and

$$
|X| \geq \frac{\sqrt{\operatorname{minclass}(G)}}{|S|} \geq \operatorname{minclass}(G)^{1 / 4} .
$$

It follows from the first part of the proof that $G$ is a product of fewer than $c \log |G| / \log |X|$ conjugates of $X$. Therefore $G$ is a product of fewer than $m c \log |G| / \log |X|$ conjugates of $S$ and, since $\log |X|=m \log |S|$, the result follows. 


\section{Plünnecke-Ruzsa estimates for nonabelian groups}

The following basic result in additive combinatorics is due to Plünnecke [21], [22] (see also Section 6.5 of [30]).

Theorem 4.1. Let $A$ and $B$ be finite sets in an abelian group $G$ and suppose that $|A B| \leq K|A|$ where $K$ is a positive number. Then for any positive integer $m$ there exists a nonempty subset $X$ of $A$ such that

$$
\left|X B^{m}\right| \leq K^{m}|X|
$$

In particular, $\left|B^{2}\right| \leq K|B|$ implies that $\left|B^{m}\right| \leq K^{m}|B|$ for $m=1,2, \ldots$.

The last statement ("In particular...") is called the Doubling Lemma; it does not hold for nonabelian groups, however, as we saw in Lemma 2.1, there are useful analogues in this context due to Helfgott, Petridis, Ruzsa and Tao [7], [20], [25], [26], [29]. Petridis also proved the following lemma, which is Proposition 2.1 of [20].

Lemma 4.2. Let $X$ and $B$ be finite sets in a group. Suppose that

$$
\frac{|X B|}{|X|} \leq \frac{|Z B|}{|Z|}
$$

for all $Z \subseteq X$. Then, for all finite sets $C$,

$$
|C X B| \leq \frac{|C X||X B|}{|X|} .
$$

Using this lemma we can extend Plünnecke's theorem to normal subsets of nonabelian groups. The statement and proof mimic Theorem 3.1 of [20], which is a stronger version of Theorem 4.1 .

Theorem 4.3. Let $A$ and $B$ be finite sets in a group $G$ with $B$ normal in $G$. Suppose that $|A B| \leq K|A|$ for some positive number $K$. Then there exists a nonempty subset $X$ of $A$ such that

$$
\left|X B^{m}\right| \leq K^{m}|X|
$$

for $m=1,2, \ldots$ In particular, $\left|B^{2}\right| \leq K|B|$ implies that $\left|B^{m}\right| \leq K^{m}|B|$ for $m=1,2, \ldots$

Proof. We proceed by induction on $m$. First choose $X \subseteq A$ such that

$$
\frac{|X B|}{|X|} \leq \frac{|Z B|}{|Z|}
$$

for all $Z \subseteq A$. Then

$$
|X B| \leq|X| \frac{|A B|}{|A|} \leq K|X|,
$$

so the result is true for $m=1$. 
Now suppose that $\left|X B^{m}\right| \leq K^{m}|X|$ for some positive integer $m$. Normality of $B$ implies that $\left|X B^{m+1}\right|=\left|B^{m} X B\right|$, and then Lemma 4.2 gives

$$
\left|X B^{m+1}\right|=\left|B^{m} X B\right| \leq \frac{\left|B^{m} X\right||X B|}{|X|} \leq K^{m+1}|X| .
$$

This verifies the inductive step, and completes the proof of the theorem.

Following an argument of Petridis (see the proof of Theorem 1.2 of [20]) we observe that the Plünnecke-Ruzsa estimates (Corollary 6.29 of [30]) can also be generalised using Theorem 4.3.

Corollary 4.4. Suppose that $A$ and $B$ are subsets of a group $G$, with $B$ normal in $G$, and $|A B| \leq K|A|$. Then

$$
\left|B^{m} B^{-n}\right| \leq K^{m+n}|A|
$$

for all positive integers $m$ and $n$.

Theorem 4.3 suggests that certain techniques in additive combinatorics concerning subsets of abelian groups can be applied to normal subsets of nonabelian groups. The next example - which is a consequence of Plünnecke's theorem, and generalises Corollary 2.4 of [25] - supports this suggestion.

Theorem 4.5. Let $A$ and $B$ be subsets of a group $G$ with $B$ normal in $G$, and suppose that $\left|A B^{j}\right| \leq K|A|$ for some positive integer $j$. If $m \geq j$ then

$$
\left|B^{m}\right| \leq K^{\frac{m}{j}}|A|
$$

Sketch of proof. We use the notation of Section 6.5 of [30]. Construct the $m$-tuple of directed bipartite graphs

$$
\left(G_{A, B}, G_{A B, B}, \ldots, G_{A B^{m-1, B}}\right) .
$$

This $m$-tuple is a Plünnecke graph. Now Plünnecke's theorem, Theorem 6.27 of [30], yields the result immediately.

\section{Proof of Theorem 1.5}

In this section we prove Theorem 1.5 and generalise some related results of Shalev. We will need the following theorem of Liebeck and Shalev [16].

Theorem 5.1. There exists an absolute positive constant a such that if $G$ is a finite simple group and $S$ is a nontrivial normal subset of $G$, then $G=S^{m}$, where $m \leq$ $a \frac{\log |G|}{\log |S|}$. 
Proof of Theorem 1.5. Let $a$ be the absolute constant from Theorem 5.1. Choose a positive integer $b$ larger than $2 a$. Suppose first that $|S| \geq \sqrt{|G|}$. Then Theorem 5.1 implies that $G=S^{m}$ where

$$
m \leq \frac{a \log |G|}{\log |S|} \leq 2 a \leq b,
$$

and hence $S^{b}=G$.

Now suppose that $|S| \leq \sqrt{|G|}$. Then

$$
\frac{\log |S|}{a \log |G|} \geq \frac{\log |S|}{2 a(\log |G|-\log |S|)}=\frac{\log |S|}{2 a(\log (|G| /|S|)} .
$$

Theorem 5.1 implies, once again, that for some $m \leq \frac{a \log |G|}{\log |S|}$ we have $G=S^{m}$. Hence, applying Theorem 4.3 to the normal subset $S$, we see that

$$
\frac{\left|S^{2}\right|}{|S|} \geq\left(\frac{\left|S^{m}\right|}{|S|}\right)^{\frac{1}{m}} \geq\left(\frac{|G|}{|S|}\right)^{\frac{\log |S|}{a \log |G|}} \geq\left(\frac{|G|}{|S|}\right)^{\frac{\log |S|}{2 a(\log (|G| /|S|)}}=|S|^{\frac{1}{2 a}} \geq|S|^{\frac{1}{b}},
$$

and this completes the proof.

The next result is a strengthening of Theorem 7.4 of [27].

Proposition 5.2. For every $\delta>0$ there exists $\varepsilon>0$ such that for any finite simple group $G$ and subsets $A$ and $B$ of $G$ with $B$ normal in $G$ and $|A| \leq|G|^{1-\delta}$ we have

$$
|A B| \geq|A||B|^{\varepsilon} .
$$

Proof. We assume that $A$ is nonempty and $B$ is nontrivial, otherwise the result is immediate.

By Theorem 5.1, $G=B^{m}$, where $m \leq a \frac{\log |G|}{\log |B|}$. Let $K=|A B| /|A|$. Then, by Theorem 4.3, there is a nonempty subset $X$ of $A$ such that $\left|X B^{m}\right| \leq K^{m}|X|$. It follows that

$$
|G|=\left|B^{m}\right|=\left|X B^{m}\right| \leq K^{m}|X| \leq K^{m}|A| .
$$

Since $|A| \leq|G|^{1-\delta}$ and $m \leq a \frac{\log |G|}{\log |B|}$ we can rearrange this inequality to give

$$
|G|^{\delta} \leq K^{a \frac{\log |G|}{\log |B|}} .
$$

This is equivalent to $|B|^{\frac{\delta}{a}} \leq K$, which, with $\varepsilon=\frac{\delta}{a}$, is the required result.

Proposition 5.2 constitutes the expansion result for $B^{2}$ that was partially proven in Proposition 10.4 of [27]. Furthermore it goes some way towards a proof of Conjecture 10.3 of [27], although what remains is the more difficult part of the conjecture.

We can strengthen Proposition 10.4 of [27] in a different direction as follows. 
Proposition 5.3. For every $\delta>0$ and positive integer $r$ there exists $\varepsilon>0$ such that for any finite simple group $G$ of Lie type of rank $r$ and any set $S \subseteq G$ such that $|S| \leq|G|^{1-\delta}$, there exists $g$ in $G$ such that

$$
\left|S S^{g}\right| \geq|S|^{1+\varepsilon}
$$

Proof. Given $\delta>0$ and a positive integer $r$, let $\varepsilon$ be the positive constant from Theorem 1.4. Now choose any subset $S$ of $G$ such that $|S| \leq|G|^{1-\delta}$. According to Theorem 1.4, either $\left|S S^{g}\right| \geq|S|^{1+\varepsilon}$ or else $S^{3}=G$. In the former case the result is proven. In the latter case we apply Lemma 2.1 with $J=K=\left(\left|S^{3}\right| /|S|\right)^{1 / 10}$ to deduce the existence of an element $g$ of $G$ with $|S g S|>K|S|$. Then, using $S^{3}=G$ and $|G| \geq|S|^{1+\delta}$, it follows that

$$
|S g S|>\left(\frac{\left|S^{3}\right|}{|S|}\right)^{\frac{1}{10}}|S| \geq|S|^{1+\frac{\delta}{10}} .
$$

Provided that $\varepsilon$ is chosen to be smaller than $\frac{\delta}{10}$, the inequality $\left|S S^{g}\right| \geq|S|^{1+\varepsilon}$ is again satisfied.

\section{The Skew Doubling Lemma}

The next result is another analogue of the Doubling Lemma for nonabelian groups, which we call the Skew Doubling Lemma.

Lemma 6.1 (Skew Doubling Lemma). If $S$ is a finite subset of a group $G$ such that, for some positive number $K,\left|S S^{g}\right| \leq K|S|$ for every conjugate $S^{g}$ of $S$, then

$$
\left|S_{1} \ldots S_{m}\right| \leq K^{14(m-1)}|S|
$$

for $m=1,2, \ldots$, where each of $S_{1}, \ldots, S_{m}$ is any conjugate of either $S$ or $S^{-1}$.

To prove Lemma 6.1 we will use Lemma 2.1 and the following result, Ruzsa's triangle inequality [24] (see also Section 2.3 of [30]).

Lemma 6.2. Let $U, V$ and $W$ be finite subsets of a group $G$. Then

$$
\frac{\left|V W^{-1}\right|}{|U|} \leq \frac{\left|U V^{-1}\right|}{|U|} \frac{\left|U W^{-1}\right|}{|U|} .
$$

First we prove a special case of Lemma 6.1.

Lemma 6.3. Let $S$ be a finite subset of a group $G$. Suppose that $K$ is a positive number such that $\left|S S^{g}\right| \leq K|S|$ for each $g$ in $G$. Then $\left|S_{1} S_{2} S_{3}\right| \leq K^{14}|S|$, where each of $S_{1}, S_{2}$ and $S_{3}$ is any conjugate of either $S$ or $S^{-1}$. 
Proof. Choose elements $a$ and $b$ of $G$. We can apply Lemma 2.1 with $J=K$ to obtain

$$
\left|S^{3}\right| \leq K^{8}|S|
$$

Using this inequality and Lemma 6.2 (with $U=S^{-1}, V=S S$ and $W=S$ ) we obtain

$$
\frac{\left|S S S^{-1}\right|}{|S|} \leq \frac{\left|S^{-1} S^{-1} S^{-1}\right|}{|S|} \frac{\left|S^{-1} S^{-1}\right|}{|S|}=\frac{|S S S|}{|S|} \frac{|S S|}{|S|} \leq K^{9} .
$$

Using this inequality and Lemma 6.2 (with $U=S, V=S^{-1}$ and $W=S S^{-1}$ ) we obtain

$$
\frac{\left|S^{-1} S S^{-1}\right|}{|S|} \leq \frac{|S S|}{|S|} \frac{\left|S S S^{-1}\right|}{|S|} \leq K^{10} .
$$

Using this inequality and Lemma 6.2 (with $U=S^{-1}, V=S S^{-1}$ and $W=S a$ ) we obtain

$$
\frac{\left|S S^{-1} a^{-1} S^{-1}\right|}{|S|} \leq \frac{\left|S^{-1} S S^{-1}\right|}{|S|} \frac{\left|S^{-1} a^{-1} S^{-1}\right|}{|S|} \leq K^{11} .
$$

Using this inequality and Lemma 6.2 (with $U=S, V=S a S$ and $W=S^{-1} b^{-1}$ ) we obtain

$$
\frac{|S a S b S|}{|S|} \leq \frac{\left|S S^{-1} a^{-1} S^{-1}\right|}{|S|} \frac{|S b S|}{|S|} \leq K^{12} .
$$

Using this inequality and Lemma 6.2 (with $U=S, V=S^{-1}, W=S^{-1} b^{-1} S^{-1} a^{-1}$ ) we obtain

$$
\frac{\left|S^{-1} a S b S\right|}{|S|} \leq \frac{|S S|}{|S|} \frac{|S a S b S|}{|S|} \leq K^{13} .
$$

Finally, using this inequality and Lemma 6.2 (with $U=S^{-1}, V=S^{-1} a S b$ and $W=S$ ) we obtain

$$
\frac{\left|S^{-1} a S b S^{-1}\right|}{|S|} \leq \frac{\left|S^{-1} b^{-1} S^{-1} a^{-1} S\right|}{\left|S^{-1}\right|} \frac{\left|S^{-1} S^{-1}\right|}{\left|S^{-1}\right|}=\frac{\left|S^{-1} a S b S\right|}{|S|} \frac{|S S|}{|S|} \leq K^{14} .
$$

Equations (6.1), (6.2) and (6.3) imply that, given any conjugates $S_{1}, S_{2}$ and $S_{3}$ of either $S$ or $S^{-1}$, we have $\left|S_{1} S_{2} S_{3}\right| /|S| \leq K^{14}$, as required.

We need the following proposition.

Proposition 6.4. If $A$ and $B$ are finite subsets of a group $G$ such that, for some positive number $K,\left|B B^{g}\right| \leq K|B|$ for every conjugate $B^{g}$ of $B$, then

$$
\left|A B_{1} B_{2}\right| \leq K^{14}\left|A B_{3}\right|
$$

where each of $B_{1}, B_{2}$ and $B_{3}$ is any conjugate of $B$ or $B^{-1}$. 
Proof. By Lemma 6.3 we have

$$
\frac{\left|B_{3}^{-1} B_{1} B_{2}\right|}{\left|B_{3}\right|} \leq K^{14}
$$

where each of $B_{1}, B_{2}$ and $B_{3}$ is any conjugate of $B$ or $B^{-1}$. Applying Lemma 6.2 with $U=B_{3}^{-1}, V=A$ and $W=B_{2}^{-1} B_{1}^{-1}$ we obtain

$$
\frac{\left|A B_{1} B_{2}\right|}{\left|A B_{3}\right|}=\frac{\left|A B_{1} B_{2}\right|}{\left|B_{3}^{-1} A^{-1}\right|} \leq \frac{\left|B_{3}^{-1} B_{1} B_{2}\right|}{\left|B_{3}\right|} \leq K^{14},
$$

as required.

We can finally prove Lemma 6.1.

Proof of the Skew Doubling Lemma. The result holds trivially when $m=1$ and $m=$ 2. Suppose that $m \geq 3$. Apply Proposition 6.4 with $B=S, A=S_{1} \ldots S_{n-2}$, $B_{1}=B_{3}=S_{n-1}$ and $B_{2}=S_{n}$ to see that

$$
\frac{\left|S_{1} \ldots S_{n}\right|}{\left|S_{1} \ldots S_{n-1}\right|} \leq K^{14}
$$

for $n=3,4, \ldots, m$. It follows that

$$
\begin{aligned}
\frac{\left|S_{1} \ldots S_{m}\right|}{|S|} & =\left(\frac{\left|S_{1} \ldots S_{m}\right|}{\left|S_{1} \ldots S_{m-1}\right|}\right)\left(\frac{\left|S_{1} \ldots S_{m-1}\right|}{\left|S_{1} \ldots S_{m-2}\right|}\right) \ldots\left(\frac{\left|S_{1} S_{2} S_{3}\right|}{\left|S_{1} S_{2}\right|}\right)\left(\frac{\left|S_{1} S_{2}\right|}{\left|S_{1}\right|}\right) \\
& \leq\left(K^{14}\right)^{m-2} K \\
& \leq K^{14(m-1)},
\end{aligned}
$$

as required.

Using the Skew Doubling Lemma we can derive Conjecture 1.6 from Conjecture 1.1. The proof is similar to the proof of Theorem 1.5.

Proof that Conjecture 1.1 implies Conjecture 1.6. Let $c$ be the absolute constant from Conjecture 1.1. We define $b$ to be a positive integer greater than $2 c$, and $\varepsilon=1 /(28 c)$. Suppose first that $|S| \geq \sqrt{|G|}$. Then Conjecture 1.1 implies that $G=S_{1} \ldots S_{N}$, for conjugates $S_{1}, \ldots, S_{N}$ of $S$, where

$$
N \leq \frac{c \log |G|}{\log |S|} \leq 2 c<b,
$$

and hence $G$ is certainly the product of $b$ conjugates of $S$.

Now suppose that $|S| \leq \sqrt{|G|}$. Then

$$
\frac{\log |G|-\log |S|}{c \log |G|-\log |S|} \geq \frac{\log |G|-\log |S|}{c \log |G|} \geq \frac{1}{2 c} .
$$


In particular observe that

$$
c \log |G|-\log |S| \leq 2 c(\log |G|-\log |S|)=2 c \log (|G| /|S|) .
$$

Conjecture 1.1 implies, once again, that for some $N \leq \frac{c \log |G|}{\log |S|}$ we have $G=$ $S_{1} \ldots S_{N}$, for conjugates $S_{1}, \ldots, S_{N}$ of $S$. Using the Skew Doubling Lemma, Lemma 6.1, we see that there is an element $g$ of $G$ for which

$$
\begin{aligned}
\frac{\left|S S^{g}\right|}{|S|} & \geq\left(\frac{\left|S_{1} \ldots S_{N}\right|}{|S|}\right)^{\frac{1}{14(N-1)}} \\
& \geq\left(\frac{|G|}{|S|}\right)^{\frac{\log |S|}{14(c \log |G|-\log |S|)}} \\
& \geq\left(\frac{|G|}{|S|}\right)^{\frac{\log |S|}{28 c(\log (|G| /|S|))}} \\
& \geq|S| \frac{1}{28 c},
\end{aligned}
$$

and this completes the proof.

\section{References}

[1] L. Babai, N. Nikolov, and L. Pyber, Product growth and mixing in finite groups. In Proceedings of the Nineteenth Annual ACM-SIAM Symposium on Discrete Algorithms, ACM, New York 2008 248-257. Zbl 1192.60016 MR 2485310

[2] G. M. Bergman and H. W. Lenstra, Jr., Subgroups close to normal subgroups. J. Algebra 127 (1989), 80-97. Zbl 0641.20023 MR 1029404

[3] E. Breuillard, B. Green, and T. Tao, Approximate subgroups of linear groups. Geom. Funct. Anal. 21 (2011), 774-819. Zbl 1229.20045 MR 2827010

[4] W. T. Gowers, Quasirandom groups. Combin. Probab. Comput. 17 (2008), 363-387. Zbl 1191.20016 MR 2410393

[5] R. M. Guralnick and W. M. Kantor, Probabilistic generation of finite simple groups. $J$. Algebra 234 (2000), 743-792. Zbl 0973.20012 MR 1800754

[6] H. A. Helfgott, Growth and generation in $\operatorname{SL}_{2}(\mathbb{Z} / p \mathbb{Z})$. Ann. of Math. (2) 167 (2008), 601-623. Zbl 1213.20045 MR 2415382

[7] H. A. Helfgott, Growth in $\mathrm{SL}_{3}(\mathbb{Z} / p \mathbb{Z})$. J. Eur. Math. Soc. (JEMS) 13 (2011), 761-851. Zbl 1235.20047 MR 2781932

[8] M. Kassabov, A. Lubotzky, and N. Nikolov, Finite simple groups as expanders. Proc. Natl. Acad. Sci. USA 103 (2006), 6116-6119. Zbl 1161.20010 MR 2221038

[9] P. Kleidman and M. Liebeck, The subgroup structure of the finite classical groups. London Math. Soc. Lecture Note Ser. 129, Cambridge University Press, Cambridge 1990. Zbl 0697.20004 MR 1057341 
[10] V. Landazuri and G. M. Seitz, On the minimal degrees of projective representations of the finite Chevalley groups. J. Algebra 32 (1974), 418-443. Zbl 0325.20008 MR 0360852

[11] M. W. Liebeck, N. Nikolov, and A. Shalev, A conjecture on product decompositions in simple groups. Groups Geom. Dyn. 4 (2010), 799-812. Zbl 1227.20006 MR 2727665

[12] M. W. Liebeck, N. Nikolov, and A. Shalev, Groups of Lie type as products of $\mathrm{SL}_{2}$ subgroups. J. Algebra 326 (2011), 201-207. Zbl 1225.20016 MR 2746060

[13] M. W. Liebeck, N. Nikolov, and A. Shalev, Product decompositions in finite simple groups. Bull. Lond. Math. Soc. 44 (2012), 469-472. Zbl 1250.20018 MR 2966992

[14] M. W. Liebeck, C. E. Praeger, and J. Saxl, The maximal factorizations of the finite simple groups and their automorphism groups. Mem. Amer. Math. Soc. 86 (1990), no 432. Zbl 0703.20021 MR 1016353

[15] M. W. Liebeck and L. Pyber, Finite linear groups and bounded generation. Duke Math. J. 107 (2001), 159-171. Zbl 1017.20039 MR 1815254

[16] M. W. Liebeck and A. Shalev, Diameters of finite simple groups: sharp bounds and applications. Ann. of Math. (2) 154 (2001), 383-406. Zbl 1003.20014 MR 1865975

[17] A. Lubotzky, Finite simple groups of Lie type as expanders. J. Eur. Math. Soc. (JEMS) 13 (2011), 1331-1341. Zbl 1257.20016 MR 2825166

[18] N. Nikolov, A product decomposition for the classical quasisimple groups. J. Group Theory 10 (2007), 43-53. Zbl 1119.20025 MR 2288458

[19] N. Nikolov and L. Pyber, Product decompositions of quasirandom groups and a Jordan type theorem. J. Eur. Math. Soc. (JEMS) 13 (2011), 1063-1077. Zbl 1228.20020 MR 2800484

[20] G. Petridis, New proofs of Plünnecke-type estimates for product sets in groups. Combinatorica 32 (2012), no. 6, 721-733.

[21] H. Plünnecke, Eigenschaften und Abschätzungen von Wirkungsfunktionen. BMwF-GMD22, Gesellschaft für Mathematik und Datenverarbeitung, Bonn 1969. Zbl 0199.36602 MR 0252348

[22] H. Plünnecke, Eine zahlentheoretische Anwendung der Graphentheorie. J. Reine Angew. Math. 243 (1970), 171-183. Zbl 0199.36701 MR 0266892

[23] L. Pyber and E. Szabó, Growth in finite simple groups of Lie type of bounded rank. Preprint, arXiv: 1005.1858 [math.GR].

[24] I. Z. Ruzsa, Sums of finite sets. In Number theory: New York Seminar 1991-1995. Springer-Verlag, New York 1996, 281-293. Zbl 0869.11011 MR 1420216

[25] I. Z. Ruzsa, Sumsets and structure. In Combinatorial number theory and additive group theory, Adv. Courses Math. CRM Barcelona, Birkhäuser Verlag, Basel 2009, 87-210. Zbl 1221.11026 MR 2522038

[26] I. Z. Ruzsa, Towards a noncommutative Plünnecke-type inequality. In An irregular mind, Bolyai Soc. Math. Stud. 21, János Bolyai Math. Soc., Budapest 2010, 591-605. Zbl 1221.11027 MR 2815615

[27] A. Shalev, Word maps, conjugacy classes, and a noncommutative Waring-type theorem. Ann. of Math. (2) 170 (2009), 1383-1416. Zbl 1203.20013 MR 2600876 
[28] A. Stein, $1 \frac{1}{2}$-generation of finite simple groups. Beiträge Algebra Geom. 39 (1998), 349-358. Zbl 0924.20027 MR 1642676

[29] T. Tao, Product set estimates for non-commutative groups. Combinatorica 28 (2008), 547-594. Zbl 1254.11017 MR 2501249

[30] T. Tao and V. Vu, Additive combinatorics. Cambridge Stud. Adv. Math. 105, Cambridge University Press, Cambridge 2006. Zbl 1179.11002 MR 2289012

Received May 15, 2012; revised October 6, 2012

N. Gill, Department of Mathematics and Statistics, The Open University, Milton Keynes, MK7 6AA, United Kingdom

E-mail: nick.gill@open.ac.uk

L. Pyber, A. Rényi Institute of Mathematics, Hungarian Academy of Sciences, P.O. Box 127, 1364 Budapest, Hungary

E-mail: pyber@renyi.hu

I. Short, Department of Mathematics and Statistics, The Open University, Milton Keynes, MK7 6AA, United Kingdom

E-mail: ian.short@open.ac.uk

E. Szabó, A. Rényi Institute of Mathematics, Hungarian Academy of Sciences, P.O. Box 127, 1364 Budapest, Hungary

E-mail: endre@renyi.hu 